\title{
TURBULENCIAS DESDE EL MAR: CHILE Y BOLIVIA*
}

\section{Turbulences from the sea: Bolivia and Chile}

\author{
Loreto Correa Vera ${ }^{* *}$ \\ Viviana García Pinzón ${ }^{\star * *}$
}

Recibido: 23 de octubre de 2012. Aprobado: 7 de marzo de 2013.

\section{RESUMEN}

En el primer semestre de 2011, Bolivia tomó la decisión de cambiar su estrategia de relacionamiento bilateral hacia Chile al crear la Dirección de Reivindicación Marítima, en la perspectiva de llevar ante tribunales internacionales su reclamo por una salida al océano Pacífico. Así, mientras para el Gobierno boliviano el diálogo no es excluyente con la búsqueda de otros espacios para zanjar las diferencias con Chile, desde la perspectiva del Gobierno chileno las dos alternativas lo son. Bajo este contexto, el artículo aborda la dinámica y las turbulencias de la relación chileno-boliviana en las últimas dos décadas. El análisis evidencia las fuentes de cooperación y conflicto entre los dos países y plantea los posibles escenarios a futuro.

PALABRAS CLAVE: Chile, Bolivia, reivindicación marítima

* Este artículo forma parte del proyecto "La agenda institucional del tema marítimo en las relaciones chilenobolivianas: 2000-2010. Pertinencia, orientación y trayectoria”. DICYT No 031094MR de la Universidad de Santiago de Chile y del proyecto "Gobernabilidad, desarrollo y seguridad en las zonas extremas del norte y sur de Chile a comienzos del siglo XXI”. Proyecto Fondecyt No 1120405. Academia Nacional de Estudios Políticos y Estratégicos. Se agradece la colaboración de Cinthia Avellaneda Vera en la revisión de este texto.

* Academia Nacional de Estudios Políticos y Estratégicos de Chile, Santiago, Chile. Correo electrónico: loretocorreav@gmail.com

*** Universidad Nacional de Colombia, Facultad de Derecho, Ciencias Políticas y Sociales. Correo electrónico: vegarcnap@gmail.com 


\begin{abstract}
During the first half of 2011, the Bolivian government decided to change its bilateral relationship strategy with Chile through the creation of the Maritime Revindication Department, under the perspective of bringing to the international tribunals their claim for regaining access to the Pacific Ocean. For the Bolivian government dialogue is not the sole strategy, and thus does not limit them from searching for other spaces in which to resolve their differences with Chile, while from the perspective of the Chilean government, the intervention of the international tribunals goes against the possibility of dialogue. Within this context, the article discusses the dynamics and the turbulences of the Bolivian-Chilean relationship over the last two decades. The analysis shows the sources of cooperation and conflict between these two countries and proposes possible future scenarios.
\end{abstract}

KEYWORDS: Chile, Bolivia, Landlocked countries

\title{
I. INTRODUCCIÓN
}

Las dinámicas de relación vecinal entre los países del continente han sido a menudo conflictivas, complejas, a tal punto que ningún país puede afirmar que sus vínculos con todos sus vecinos son fáciles de conducir. Este fenómeno no es original ni privativo de América Latina. Desde las guerras de la independencia, el legado de desconfianzas entre las élites, encausado a través de ciclos de otras guerras (Triple Alianza, del Pacífico, del Chaco, etc.), sumadas a los resabios del caudillismo, el populismo y en el último cuarto del siglo XX, el legado de los gobiernos de facto, contribuyeron a cimentar susceptibilidades.

Desde la formación republicana, las relaciones de Chile con sus vecinos han estado atravesadas por la persistencia de los conflictos. Una ocupación (1824) y dos guerras (1836 y 1879) afectan a Chile, Perú y Bolivia de manera vinculante. Aclarados los límites, definidos los tratados territoriales (1904 con Bolivia y 1929 con Perú), Chile creció notoriamente respecto de su formación territorial independentista, principalmente debido a disputas territoriales que se remontan a finales del siglo XIX. Estas diferencias han permanecido y, entrados ya en el siglo XXI, continúan siendo factores claves en las relaciones vecinales. 
En el caso de Perú, se encuentra el diferendo planteado por este país a Chile en relación a la delimitación marítima de una zona de $37.900 \mathrm{~km}$ en el océano Pacífico. Tras la demanda interpuesta por parte del Estado peruano, la decisión sobre esta disputa territorial se encuentra en manos de la Corte Internacional de Justicia en La Haya. Con Argentina, el desacuerdo respecto a la delimitación territorial sobre territorios patagónicos (Conflicto del Beagle) atravesó el siglo XX, llegando a un punto crítico en 1978; luego de la mediación de la Santa Sede, el Tratado de Paz y Amistad de 1984 puso fin al desacuerdo. Finalmente, la relación con Bolivia ha estado caracterizada por el tema de la mediterraneidad boliviana y su demanda por una salida al océano Pacífico. Ante la ausencia de una solución definitiva por la reivindicación marítima, los dos países mantienen rotas sus relaciones diplomáticas desde 1978. La consideración de las relaciones vecinales priorizando las perspectivas militares y estratégicas, configuró una visión del otro en términos de amenaza, alimentando percepciones negativas y por extensión, relaciones conflictivas y de juego de suma cero.

Sin embargo, desde la década de 1970 la política mundial ha cambiado. Las transformaciones en el sistema internacional, con una mayor importancia de las temáticas económicas y el aparente declive del uso de la fuerza y de la importancia de los temas de seguridad, implican la consideración de nuevas dinámicas en las relaciones interestatales y la emergencia de nuevos actores en la arena internacional. Celestino del Arenal (1994) señala que, a partir del término de la guerra fría, uno de los cambios más importantes que ha ocurrido en materia internacional se relaciona con

el debilitamiento del rol y significado del Estado, y la aparición de nuevos actores, tanto intergubernamentales como no gubernamentales, de las relaciones internacionales, [y que] el sistema internacional ha perdido, dado el carácter estatocéntrico anterior. La marcada diferencia entre la política doméstica y política internacional desaparece. El fenómeno de la interdependencia y la necesidad de atender a las demandas de desarrollo económico y social ha obligado al Estado a abrirse cada vez más al exterior, lo que ha incrementado dicha interdependencia y restringido su margen de autonomía. Todo ello hace que ya no sea posible separar la política interna y la política internacional y que el comportamiento internacional del Estado no pueda explicarse en términos políticos y militares... (1994:32-3).

En el caso de Chile, los cambios en la esfera internacional y en el contexto regional, así como las transformaciones en la política doméstica tras el retorno a la democracia (Wilhelmy y Durán 2003; Quezada 2010), enmarcan un cambio en las relaciones con los países vecinos. Así, los vínculos con Argentina y Perú reflejan un permanente interés por 
mejorar paulatinamente tanto el comercio y la balanza comercial, así como por resolver las dificultades mencionadas en el marco de un diálogo abierto y no exento de polémicas, dificultades políticas, presidenciales y hasta personales entre las autoridades de Estado. La relación con Bolivia también se ha visto compelida a modificar su tradicional statu quo y particularmente, obligada a ampliar y superar las fórmulas "realistas" de entendimiento mutuo. Se advierte que a partir de la década de 1990 hay un cambio progresivo en el relacionamiento de los dos países. Algunas veces, este cambio ha sido valorado positivamente desde la agenda política; otras, de forma negativa -criticada ácidamente por los medios y la opinión de especialistas y funcionarios de Estado involucrados en la relación vecinal.

Dicho acercamiento está apuntalado en un contexto de mayor integración e interdependencia regional, los intereses económicos (el comercio y los recursos energéticos) y las dinámicas migratorias y demográficas. Su expresión más acabada y concreta la constituye la Agenda de los Trece Puntos, que marca la hoja de ruta de la relación bilateral. Su formulación, en 2006, por parte de Michelle Bachelet en Chile y Evo Morales en Bolivia, inauguró - particularmente en Bolivia y contra todo pronóstico por el histórico discurso masista- una nueva forma de representación política en lo interno y una nueva vertiente de relacionamiento bilateral entre los dos países centrada en la construcción de las confianzas mutuas.

Dentro de los 13 aspectos formulados, se encuentra el de la mediterraneidad boliviana, tema que evolucionó a partir de la fijación de una agenda sin exclusiones. La agenda se configuró como un mecanismo bilateral para la búsqueda de un acuerdo sobre este tema; un espacio que privilegia el diálogo "cara a cara" entre las partes involucradas, dejando de lado la participación de terceros países o de tribunales internacionales. Lo anterior, dada la historia de desavenencias en torno a la cuestión marítima, marca una particular importancia en la historia de la relación chileno-boliviana. Sin embargo, a comienzos de 2011, hubo un nuevo giro en la dinámica de la relación: el gobierno boliviano, en cabeza de Evo Morales, decidió crear la Dirección de Reivindicación Marítima y anunció su intención de demandar a Chile ante la Corte Internacional de Justicia de La Haya. Desde el punto de vista boliviano, un eventual juicio no es excluyente con la prosecución del diálogo bilateral; mientras que para Chile los dos escenarios son excluyentes y el acudir a un Tribunal de Justicia Internacional está fuera de lugar, en la medida en que, desde el punto de vista del Gobierno chileno, el Tratado de 1904 resolvió el tema territorial en todas sus dimensiones. 
En este contexto surgen las preguntas que motivan el presente artículo: ¿cómo explicar el acercamiento bilateral? ¿Cuáles son las temáticas que, planteadas en la Agenda de los Trece Puntos, configuran la relación chileno-boliviana? ¿Cuáles fueron las motivaciones del Gobierno boliviano para romper la dinámica bilateral reflejada en la agenda y optar por la idea de plantear la intervención de los tribunales internacionales?

El objetivo del presente trabajo es identificar las temáticas e incentivos que dan cuenta de los cambios de una relación vecinal, pasando de una interacción marcada principalmente por la conflictividad y la ignorancia mutua, a una de acercamiento, en la que se ha avanzado por trayectorias de cooperación, en la medida en que se ha ido fortaleciendo la construcción de una confianza mutua. Ello, a pesar de la continuidad de las diferencias y desacuerdos, marcados principalmente por la demanda boliviana de salida al mar. A partir de la revisión de documentos oficiales y prensa ${ }^{1}$ de los dos países, son analizadas las dinámicas de la relación bilateral durante la última década (2002-2012).

Nuestra hipótesis es que el cambio en la dinámica chileno-boliviana se debe a la creciente institucionalización en la relación, la cual ha estado favorecida por factores de estructura y contexto. Estos factores serían la mayor interdependencia e integración regional, densidad temática en la relación bilateral, las transformaciones en la política doméstica, en particular, el retorno a la democracia en Chile y la estrategia de reinserción internacional que sucede a este período. No obstante, la cooperación no implica -hay que remarcarlo- la armonía de intereses (Keohane 1993), de modo tal que persisten los factores de conflicto entre los dos países: la demanda marítima boliviana y la desconfianza por parte de Chile frente a la inestable dinámica de la política doméstica boliviana. La política exterior chilena hacia Bolivia ha operado de manera reactiva en función de la mayor o menor expectativa de confianza que la política interna de este país ofrece a Chile. En períodos de estabilidad relativa, la prioridad para Chile fue avanzar en la vinculación, y en períodos de inestabilidad replegarse. Con todo, el centro de la relación para Chile es "llevarse bien con el vecino", mientras que para Bolivia, el punto clave es avanzar en el tema marítimo, hasta el punto de recuperar una salida soberana al mar. Este incordio traspasa la relación vecinal y en buena medida explica el carácter zigzagueante de la misma.

En la primera parte del artículo son abordados los elementos de la relación bilateral que han dado lugar a un acercamiento y la apertura a una vinculación más cooperativa. En el segundo apartado, se profundiza en el contexto del surgimiento y características de la Agenda de los Trece Puntos. En tercer lugar, se analizan el cambio en la estrategia de reivindicación marítima por parte de Bolivia en el período más reciente y sus impli-

1 Habitualmente interlocutora de los temas binacionales. 
caciones en la relación con Chile. Finalmente, se presentan algunas conclusiones, para dar cuenta del devenir de la relación chileno-boliviana, a partir de la coyuntura actual.

\section{LA AGENDA COMÚN: DENSIDAD TEMÁTICA, INTERESES COMPARTIDOS Y COOPERACIÓN}

Las relaciones diplomáticas entre Chile y Bolivia están formalmente rotas desde 1978. No obstante, han existido espacios de interacción entre los dos países, a partir de los que se han buscado mecanismos para el desarrollo de iniciativas en base a los intereses conjuntos. Los avances y retrocesos en este campo han estado condicionados tanto por el entorno internacional y regional como por la política interna de cada país. Tras el retorno a la democracia en Chile, el primer gobierno de la transición, encabezado por Patricio Aylwin, tuvo como prioridad la "reinserción internacional" del país, entendida como

un proceso de positiva aceptación externa de la recuperación de las tradiciones democráticas y el comienzo de la tarea de enfrentar las consecuencias políticas, jurídicas y humanitarias del período autoritario. Ello puso fin en lo esencial a la situación de impugnación que gravitaba sobre el gobierno anterior, disminuyendo la vulnerabilidad política internacional del país, permitiendo al mismo tiempo recuperar gradualmente una mayor presencia e influencia en los niveles global, regional y bilateral de su política exterior (Wilhelmy y Durán 2003:280).

Según Quezada (2010), los ejes que articularon la estrategia de inserción fueron la concertación política y la profundización de la integración económico-comercial. Respecto a lo primero, la diplomacia chilena se reactivó en foros multilaterales. A ello se sumó la participación en cumbres, conferencias y visitas oficiales. Con estas premisas cumplidas, la dimensión regional comenzó a desarrollarse particularmente en lo que la Cancillería denominó la definición de prioridades en la política exterior.

El eje de integración económico-comercial se expresó en una política de regionalismo abierto, caracterizada por la apertura unilateral sobre la base de la reducción de aranceles y las negociaciones comerciales en espacios multilaterales, regionales y bilaterales.

De esta manera, a diferencia de lo ocurrido en otros países latinoamericanos, en Chile, los gobiernos de la Concertación no tuvieron que diseñar un nuevo modelo de gestión 
económica para adaptarlo a los requerimientos del mundo de la posguerra fría que comenzaba a diseñarse; sólo optaron por darle continuidad a la estrategia implementada durante el régimen militar (Fazio 1997:76).

Los principales instrumentos para ello han sido los Tratados de Libre Comercio y Acuerdos de Complementación Económica.

Otro de los puntos que forman parte de la estrategia de reinserción chilena se aboca al acercamiento y mejora en las relaciones vecinales. La identificación de los asuntos pendientes (en su mayoría disputas limítrofes), y su solución, permitiría impulsar el desarrollo de iniciativas en ámbitos de interés común, particularmente el intercambio comercial, la integración vial y la cooperación energética. El contexto de regreso a la democracia y las prioridades de la política exterior chilena posibilitaron un acercamiento en sus relaciones con Bolivia y la puesta en marcha para abordar temáticas que interesan a los dos países, salvo el tema de la salida al océano Pacífico reclamada por Bolivia.

Entre tanto, los gobiernos de Bolivia desde 1985 hasta 2005 adoptaron una estrategia de inserción mediante la apertura de una mayor inversión extranjera e intercambio comercial, con medidas dirigidas al estímulo a las exportaciones (enfatizando en estrategias de incentivos fiscales) y la flexibilización de importaciones (Muriel y Barja 2006; Seoane 2008). Así, Bolivia adhirió al GATT (General Agreement on Tariffs and Trade/ Acuerdo General sobre Comercio y Aranceles) y suscribió el acuerdo que dio lugar a la creación de la Organización Mundial del Comercio (OMC), se asoció al Mercosur a través de una Zona de Libre Comercio y se integró a la Iniciativa para el Desarrollo de la Infraestructura Regional Sudamericana (IIRSA). Entrado en el siglo XXI, el hecho de ser el país con las segundas mayores reservas de gas en Sudamérica en el marco de la Ley de Capitalización (Correa 2007), constituyó un incentivo para que el Gobierno boliviano enfatizase la búsqueda de nuevas asociaciones y acuerdos comerciales para promover el crecimiento del sector de los hidrocarburos. A partir de los descubrimientos de reservas y de la capitalización boliviana se abrió un amplio abanico de oportunidades de mercados. Por ello, es que entre el 2000 y 2003 se gestó la posibilidad de abrir un mercado de exportación de gas a California. Como señala Orias (2002), a través del gas se abrió una nueva posibilidad de inserción internacional para Bolivia, con el potencial de incrementar su poder político relativo y de ser el país eje en la integración energética de la región.

Así, el contexto regional, los modelos de inserción y desarrollo económico, la densidad temática, y la necesidad de abordar las problemáticas conjuntas, fueron dando lugar a un cambio en la relación bilateral y el acercamiento, a pesar de la persistencia del 
mayor e histórico factor de conflicto entre los dos países: la mediterraneidad boliviana. El tema económico -particularmente los intereses comerciales y energéticos- fue la base sobre la que se apuntaló el mayor acercamiento entre Chile y Bolivia. Esto dio paso al desarrollo de una serie de instituciones y organismos para abordar temas como la seguridad, la infraestructura y las migraciones, entre otras, como veremos a continuación.

\section{Relaciones comerciales: el ACE $N^{\circ} 22$}

En abril de 1993 fue firmado el Acuerdo de Complementariedad Económica (ACE) entre Bolivia y Chile, a través del llamado ACE No 22 que entró en vigencia el 7 de julio de 1993. El objetivo del acuerdo era crear tanto un vínculo comercial más estrecho como generar un marco para la cooperación bilateral. Por ello, el ACE es representativo como instrumento comercial, pero también político. En materia comercial, el ACE comprende tres esquemas distintos de preferencias arancelarias:

1. Preferencias arancelarias del 100 por ciento otorgadas de Chile a Bolivia, sin reciprocidad, para importaciones originarias de este último. Los productos incluidos se encuentran en el Anexo I del Acuerdo y se trata de 32 ítems.

2. Liberación de los gravámenes de las importaciones a 254 productos listados en los Anexos II y III del ACE. El anexo II comprende preferencias otorgadas de Bolivia a Chile para 153 productos y el Anexo III de Chile a Bolivia en 201 ítems.

3. Consolidación de las preferencias arancelarias establecidas en el Acuerdo Parcial No 27 de 1983.

En total, las preferencias arancelarias establecidas en el ACE No 22 comprenden 360 productos. En el marco del acuerdo, fue constituida una comisión administradora, la cual tiene como misión velar por el cumplimiento de las disposiciones del acuerdo, evaluar su aplicación y acordar la inclusión de nuevos productos.

A dos décadas de que el acuerdo fue suscrito, la evolución fue la siguiente: 
Tabla No 1

Intercambio comercial bilateral (1990-2009) en millones de dólares

\begin{tabular}{|c|c|c|c|c|}
\hline Año & $\begin{array}{c}\text { Exportaciones } \\
\text { chilenas }\end{array}$ & $\begin{array}{c}\text { Importaciones } \\
\text { desde Bolivia }\end{array}$ & $\begin{array}{c}\text { Intercambio } \\
\text { comercial }\end{array}$ & $\begin{array}{c}\text { Balanza comercial } \\
\text { favorable a Chile }\end{array}$ \\
\hline 1990 & 73.249 & 21.253 & 94.502 & 51.996 \\
\hline 1991 & 112.481 & 19.489 & 131,97 & 92.992 \\
\hline 1992 & 151.398 & 16.717 & 166.115 & 134.681 \\
\hline 1993 & 161.932 & 13.122 & 175.054 & 148,81 \\
\hline 1994 & 171.482 & 25.346 & 196.828 & 146.136 \\
\hline 1995 & 196.901 & 24.669 & 221,57 & 172.232 \\
\hline 1996 & 207.851 & 35.852 & 243.703 & 171.999 \\
\hline 1997 & 228.515 & 62.536 & 291.051 & 165.979 \\
\hline 1998 & 249.595 & 37.578 & 287.173 & 212.017 \\
\hline 19991 & 145,9 & 13,7 & 159,6 & 132,2 \\
\hline 2000 & 170.289 & 30.746 & 201.035 & 170.289 \\
\hline 2001 & 144.802 & 30.309 & 175.111 & 144.802 \\
\hline 2002 & 123.156 & 32.594 & 155.750 & 120.156 \\
\hline 2003 & 139,9 & 41,5 & 151,02 & 98,4 \\
\hline 2004 & 138,2 & 53,5 & 191,7 & 84,7 \\
\hline 2005 & 209,7 & 37,8 & 247,5 & 171,9 \\
\hline 2006 & 281,8 & 58,6 & 340,4 & 223,2 \\
\hline 2007 & 299,8 & 57,0 & 356,8 & 242,8 \\
\hline 2008 & 419,2 & 78,1 & 497,3 & 341,1 \\
\hline 2009 & 282,5 & 79,1 & 361,6 & 203,4 \\
\hline 2010 & 304 & 83 & 387 & 221 \\
\hline 2011 & 308 & 150 & 458 & 158 \\
\hline
\end{tabular}

Fuente: Elaboración propia en base a cifras del Banco Central de Chile,

Direconmulti, IBCE y Cadex, Bolivia.

A partir de las cifras, desde comienzos de la década de 1990, la balanza comercial entre Chile y Bolivia presenta una trayectoria creciente hasta 1997. El punto más bajo fue alcanzado en 2005 con 84 millones de dólares y en 2008 alcanzó el pick más alto en las últimas dos décadas, con poco más de 341 millones de dólares. En general, para el caso particular de las relaciones económicas con Bolivia, Chile ha mantenido siempre una balanza favorable y positiva. Pero las cifras son contundentes: el mercado boliviano solo ha llegado a representar 0,4 por ciento del total exportado al mundo desde Chile en ese año, cifra insignificante para este país, comparativamente hablando, si miramos a sus vecinos Argentina y Perú. 
A pesar de la evolución del comercio recíproco y de lo que el ACE № 22 constituyó en términos de la relación bilateral, como base y proyección para un mayor acercamiento entre Chile y Bolivia, el intercambio no ha alcanzado niveles que le permitan constituirse como un factor de entrelazamiento o interdependencia significativo entre los dos países, tanto así que Bolivia "desistiese de sus reclamaciones o para generar los incentivos suficientes para que el gobierno chileno considerara en términos reales la cesión de una franja de su territorio como las autoridades bolivianas lo han propuesto" (Deustua 2004).

\section{LA DINÁMICA FRONTERIZA: FACTORES PARA LA INSTITUCIONALIZACIÓN DE LA RELACIÓN BILATERAL}

Aunque la existencia del conflicto estratégico en torno a la salida al mar ha fragmentado la relación entre Chile y Bolivia, la cuestión limítrofe, la infraestructura, el transporte, el problema del narcotráfico y del contrabando de vehículos, así como los flujos migratorios, son temáticas obligadas en torno a las cuales se han generado un conjunto de mecanismos y canales de diálogo que moldearon la relación bilateral y promovieron la confianza mutua.

A través de un conjunto de instancias hay una institucionalización en la vinculación entre Chile y Bolivia, que permitieron un avance en la relación bilateral, de manera paralela a la persistencia del conflicto por la salida al océano Pacífico y las aguas de los ríos Silala y Lauca.

Algunas de dichas instancias son:

1. Mecanismos de consultas políticas.

2. Grupo de trabajo de asuntos bilaterales.

3. Comité de fronteras.

4. Grupo Técnico Mixto sobre infraestructura (GTM) y Acuerdo sobre el Transporte Internacional Terrestre del Cono Sur (ATIT), y

5. Comisión mixta del acuerdo sobre control, fiscalización y represión del tráfico ilícito de estupefacientes. 


\section{La Agenda de los Trece Puntos: contexto y contenido}

Tras su elección como Presidente de Bolivia, en 2006, Evo Morales buscó un acercamiento a Chile. El entonces presidente de Chile Ricardo Lagos fue invitado a la ceremonia de posesión presidencial. La prensa boliviana (Los Tiempos 2006) registró con optimismo los hechos de aquel momento, calificando la visita de Lagos como un "hecho histórico" (La Razón 2006a).

La presencia del presidente de Chile Ricardo Lagos en el país ha causado una buena impresión en el país [...]. Primero que nada esa asistencia abre un camino para mejorar las relaciones bilaterales, por más que el presidente Lagos permanecerá en el mando de su nación hasta marzo (El Deber 2006).

Posteriormente, Morales fue invitado a la ceremonia de posesión presidencial de Michelle Bachelet. Otro gesto calificado como histórico y conducente a un cambio en la relación bilateral:

es histórica no sólo por el hecho de que será la primera visita oficial de un presidente boliviano a Santiago, sino porque podría suponer el punto de inflexión en la soterrada "guerra fría" que han mantenido ambos países durante las últimas décadas (La Razón 2006b).

En el marco de esta invitación, los dos presidentes sostuvieron una reunión, que según el vicepresidente boliviano, Álvaro García Linera, sería el inicio de un "proceso de largo aliento" en la relación bilateral (La Voz 2006a).

Una de las críticas que se escucha en Bolivia sobre el tema marítimo es, precisamente, la poca seriedad y consistencia que han tenido todas las administraciones anteriores en sus relaciones internacionales, lo que Evo Morales pretende corregir fijando una agenda de trabajo con objetivos fijos en el tiempo y donde obviamente el tema marítimo será un punto relevante (La Voz 2006b).

Asimismo, el gobierno chileno se mostró dispuesto para el establecimiento de canales de diálogo con Bolivia para lograr una nueva dinámica en la relación vecinal. Pactada en junio del 2006 por el presidente boliviano Evo Morales y la entonces presidenta de Chile Michelle Bachelet y retomada el 2010 por el gobierno de Sebastián Piñera, la Agenda de los Trece Puntos planteaba y consolidaba la primera hoja de ruta entre los dos Estados. 
Esta agenda aborda:

\section{Primer Punto: Desarrollo de la Confianza Mutua}

La confianza mutua se ha constituido en el pilar fundamental de la relación. Su principal logro ha sido impulsar y desarrollar múltiples acciones en forma continua, lo que ha ampliado los espacios de diálogo a nivel bilateral y ha permitido un mayor acercamiento entre ambos países.

En este ámbito resaltan los sucesivos encuentros y reuniones a nivel presidencial, de cancilleres, otros ministros de Estado y autoridades de gobierno, así como el desarrollo de actividades donde se han iniciado y estrechado los contactos entre distintos sectores de la sociedad, tales como Parlamentos, Fuerzas Armadas, organizaciones sociales, representantes empresariales, medios de comunicación, autoridades universitarias, del mundo de la cultura, mujeres líderes, historiadores, cientistas políticos, académicos e intelectuales. La alta densidad de encuentros presidenciales en los últimos años no tuvo precedentes o similitud con otro país.

\section{Segundo Punto: Integración Fronteriza}

Para efectos de lograr la más amplia integración fronteriza fueron creados los Comités de Frontera, más tarde Comité de Frontera e Integración (2009). Dicho mecanismo fue establecido en 1997 y ha tenido como objeto promover la facilitación, integración y coordinación fronteriza, además de contribuir a un mejor conocimiento de nuestros países y zonas fronterizas y a la consolidación de un diálogo más constructivo y de mayor contenido real. Ambos países tienen un programa de trabajo que confirma a estos comités como un foro válido para vincular a las regiones de Arica y Parinacota, Tarapacá y Antofagasta con los departamentos vecinos de La Paz, Oruro y Potosí en Bolivia.

En el plano de la integración fronteriza, destacan asimismo los controles integrados de frontera, modalidad que se encuentra en fase aun de aplicación, desde el 2006 en Visviri (Dirección de Fronteras y Límites 2012a); y Colchane-Pisiga que une la ciudad boliviana de Oruro con Iquique a través de la carretera internacional A-55 (Dirección de Fronteras y Límites 2012b). El complejo, acondicionado para control integrado, es el paso Chungará-Tambo Quemado. El Complejo Chungará, llevado a cabo bajo licitación privada, significó una inversión de aproximadamente 11 millones de dólares (Dirección de Fronteras y Límites 2012b). 


\section{Tercer Punto: Libre Tránsito}

Fundamentado en el Tratado de 1904 e instrumentos posteriores, es una de las bases de la relación con Bolivia. Con ocasión del proceso de concesión portuaria en Antofagasta y Arica, el libre tránsito está presente en el marco de la modernización de puertos chilenos de la actual década y ofrece un espacio de trabajo sustancial a desarrollar entre los dos países para profundizar o perfeccionar el Tratado de 1904, dejando a Bolivia en un mucho mejor pie para sus exportaciones.

En los puertos de Arica y Antofagasta, la carga boliviana goza de gratuidad por un año calendario, en cuanto al almacenamiento de importaciones, y de 60 días en el caso de las exportaciones. La gratuidad en Antofagasta rige en el área administrada por la Empresa Portuaria Antofagasta y en Portezuelo. La habilitación del puerto de Iquique data desde el 2008.

\section{Cuarto Punto: Integración Física}

Chile y Bolivia establecieron el Grupo Técnico Mixto (GTM), en mayo 2002, para orientar prioridades en obras viales concertadas, a través de los ministerios de Obras Públicas y Cancillería. Entre las principales iniciativas de integración física, está el Corredor Interoceánico Central, que conecta Chile, Bolivia y Brasil por vía terrestre, un hito trascendente en la conectividad física continental. En el ámbito bilateral, destaca la rehabilitación y remediación de la vía férrea de la sección chilena del Ferrocarril Arica-La Paz.

\section{Quinto Punto: Complementación Económica}

Como ya se ha afirmado, el intercambio comercial con Bolivia se da en el contexto del ACE No 22 y acuerdos complementarios (ProChile 2009:2).

\section{Sexto Punto: Tema Marítimo}

Chile tiene la disposición a considerar, con las modalidades y en los tiempos que correspondan, fórmulas que posibiliten un perfeccionamiento del acceso de Bolivia al océano Pacífico, lo que requiere avanzar en el proceso de construcción de confianzas y los necesarios consensos internos. No obstante, la posición de la política exterior chilena se mantiene en una perspectiva legalista, desde la cual no se considera como una vía realista la revisión del Tratado de 1904 o la cesión de territorio con soberanía. Asimismo, las autoridades chilenas han sido claras en no aceptar la intervención de terceros países, 
como tampoco el reconocimiento de competencia alguna de organismos internacionales en esta materia.

Con ocasión de la ceremonia histórica del Día del Mar, el 23 de marzo de 2011, el Gobierno boliviano decidió "forzar" la relación, anunciando la creación de la Dirección de Reivindicación Marítima y afirmó su intención de poner ante la consideración del Tribunal Internacional de La Haya el desacuerdo con Chile en torno a la salida al océano Pacífico. La reacción inmediata del Gobierno chileno fue destacar que la opción de apelar a un tribunal internacional iba en desmedro del avance del diálogo y construcción de la confianza mutua.

\section{Séptimo Punto: Silala y Recursos Hídricos}

Las aguas del río Silala se encuentran en la provincia Sud Lípez de Potosí, y representan un curso de $15 \mathrm{mil} \mathrm{l/m}$, correspondientes a un aluvión por escurrimiento de aguas fluvioglaciales en la hondonada del cantón Quetena Chico, donde se localiza el nacimiento que sale a la superficie como manantial. Este aspecto le da la razón a Bolivia, en el sentido de que demuestra que geológicamente se trata de aguas inclasificables como río de curso sucesivo binacional.

El acuerdo inicial firmado en Quetena (2009 señala que se realizarán estudios y mediciones de los flujos superficiales. Se deduce que este 50 por ciento corresponde inicialmente al Estado Plurinacional de Bolivia y es de libre disponibilidad, pudiendo utilizarlas en su territorio o autorizar su captación para su uso por terceros, incluyendo su conducción a Chile. Un acuerdo de esta naturaleza generaría derechos o al menos cierta expectativa en Chile, que podría argüir que existe un reconocimiento implícito del 50 por ciento de las aguas a su favor, lo que le serviría argumentalmente en un posible arbitraje.

$\mathrm{Al}$ margen de lo anterior y de las eventuales repercusiones que ello pudiese conllevar, es importante resaltar que, por vez primera después de 1999, Bolivia ha aceptado que Chile tiene derechos en el Silala. Por otro lado, también es relevante destacar que Chile afirmó desde el inicio de las conversaciones, que no asumiría "compensaciones" históricas, así como que no sería posible concebir que sea el Estado el que pague por el agua que cruza por la frontera (lo que no tiene precedentes), sino que debieran acordarse mecanismos que permitieran a Bolivia constituir derechos en su territorio sobre aguas que puedan ser aprovechadas en nuestro país. 


\section{Octavo Punto: Instrumentos de Lucha contra la Pobreza}

Con fecha 13 de noviembre de 2007, se estableció un mecanismo formal de cooperación entre ambos países, creándose el Grupo de Trabajo Bilateral en esta área, designándose a la Agencia de Cooperación Internacional de Chile (AGCI) y al Viceministerio de Inversión Pública y Financiamiento Externo de Bolivia (VIPFE).

La primera sesión de este grupo de trabajo tuvo lugar en mayo de 2009 en La Paz, instancia en la que se diseñó un plan de trabajo bianual que incluía distintas áreas de interés recíproco, las que han marcado un positivo punto de inflexión para la cooperación bilateral. Entre ellas, becas, asistencia técnica, capacitación en gestión pública, iniciativas en el área de la salud, capacitación en la administración de bancos de sangre, entrega de asistencia técnica en nutrición, junto al Programa Mundial Alimentario y el cumplimiento con las metas ODM 4 y 5 en materia de salud materno e infantil (ProChile 2009).

\section{Noveno Punto: Seguridad y Defensa}

En junio de 2008, el Ministerio de Defensa de Chile suscribió un memorándum de entendimiento con el Ministerio de Defensa de la República de Bolivia sobre cooperación en el área, lo que constituía un hito en la generación de un clima de confianza y la institucionalización de las relaciones bilaterales sectoriales (Ministerio de Defensa 2010a; Vaca 2008). El quiebre de la relación desde el 2011 y la falta de protocolos en su firma, anuló la iniciativa, visitas, intercambios y los programas de cooperación en defensa.

Respecto del tema del desminado, la reunión de vicecancilleres realizada el 2010 dio cuenta del término de las acciones conducentes al desminado: "Jaime Ravinet y Rubén Saavedra firmaron un acta que declara Terrenos Libres de Minas a los campos minados Tambo Quemado 1 y Tambo Quemado 2, ubicados en la frontera de ambos países, a 140 kilómetros de Arica" (Comisión Nacional de Desminado 2010). La iniciativa -aún en curso- se enmarca dentro de los compromisos adquiridos por Chile a través de la Convención de Ottawa de 1997.

\section{Décimo Punto: Cooperación para el Control del Tráfico Ilícito de Drogas y de Productos Químicos Esenciales y Precursores}

Entre los avances destacan la intensificación del intercambio de información y cooperación entre las instituciones policiales y Ministerios Públicos. El Seminario en Arica sobre Cooperación Judicial, Policial y Lavado de Activos (2009) contribuyó al for- 
talecimiento de las políticas en el combate contra ese flagelo. La última reunión de la Comisión Mixta sobre Drogas se efectuó en agosto de 2010 (Ministerio de Relaciones Exteriores 2010a). En ella se acordó la realización de operaciones de control conjuntas; el mejoramiento en recursos humanos y la capacitación de los cuerpos policiales, jueces y fiscales de los dos países.

\section{Undécimo punto: Educación, Ciencia y Tecnología}

Entre las materias de interés mutuo abordadas en los últimos años, destacan el desarrollo de portales digitales educativos y la generación de becas para educación superior y ofertas de magíster y doctorados. También sobresale la convocatoria a una primera reunión de la Comisión Bilateral de Educación, Ciencia y Tecnología (MINREL 2010b).

\section{Duodécimo punto: Culturas}

En mayo de 2009 se suscribió el "Memorándum de entendimiento para un programa de intercambio cultural entre el Consejo Nacional de la Cultura y las Artes de Chile y el Ministerio de Educación y Culturas de Bolivia para los años 2009, 2010, 2011 y 2012", instrumento que constituye un hito histórico en las relaciones de cooperación cultural entre Chile y Bolivia (Ministerio de Relaciones Exteriores 2010b).

Como expresión práctica a dicho instrumento, se han desarrollado diversas iniciativas culturales, entre las que cabe destacar encuentros entre gestores culturales de ambos países en distintas disciplinas, la suscripción de un convenio de cooperación entre la Cinemateca de Chile y la Cinemateca de Bolivia, además de las sucesivas participaciones de Chile en la Feria Internacional del Libro de La Paz, en la cual Chile fue su invitado de honor en la versión 2007 y Bolivia en Chile en su versión 2011.

Sin embargo, en esta materia existe un déficit, que obedece al recambio de élites con el ingreso de Morales al poder, y que conectaba al mundo académico boliviano con el sector intelectual chileno. La intelectualidad, renovada a partir de los movimientos sociales y la coalición del MAS (Movimiento al Socialismo) en Bolivia, carece de interlocutores interesados en profundizar mayormente con Chile. En reemplazo, se han realizado otras iniciativas impulsadas por la Cancillería, como las visitas de líderes de opinión y agendas de trabajo compartidas en relación a la percepción mutua. 


\section{Decimotercer punto: Otros Temas}

Seguridad social, diálogo multilateral, contactos interparlamentarios y temas jurídicos y consulares, han sido ámbitos con una actividad sostenida. Este aspecto es aún complementario dentro de la agenda, pero evidencia la voluntad de las partes por dialogar todos los temas y manejar la relación de manera flexible.

En suma, la agenda fue clave dentro de la relación bilateral en el período reciente, fruto del acercamiento paulatino de las últimas dos décadas y a la que subyace la idea de que a través del diálogo es posible gestionar y resolver los asuntos y conflictos existentes en la relación chileno-boliviana. No obstante, luego de cinco años de que fuera establecida, de reuniones y encuentros bilaterales desarrollados bajo su ámbito, desde la perspectiva del Gobierno boliviano el marco establecido se mostró insuficiente para el posicionamiento y resolución de su mayor demanda y fuente de continuo desacuerdo: los recursos hídricos, en general, y la mediterraneidad de Bolivia, en particular.

\section{LA DEMANDA BOLIVIANA POR UNA SALIDA AL PACÍFICO}

La cuestión territorial en relación con los recursos hídricos (mediterraneidad boliviana y fuentes hídricas) atraviesa la relación de Chile y Bolivia, constituyendo la mayor fuente de conflicto. A partir de la aceptación del Gobierno chileno de un diálogo sin exclusiones, la Agenda de los Trece Puntos se presentó como la vía a través de la cual sería posible llegar a una resolución definitiva sobre el tema.

Sin embargo, con ocasión de la ceremonia histórica del Día del Mar, el 23 de marzo de 2011, el gobierno boliviano de Evo Morales anunció la creación de la Dirección de Reivindicación Marítima y su intención de presentar ante cortes internacionales el desacuerdo con Chile en torno a la salida al océano Pacífico. Según el presidente Evo Morales y su canciller David Choquehuanca, la eventual demanda no excluye el camino de diálogo emprendido con Chile, materializado en la denominada Agenda de los Trece Puntos. La reacción inmediata del Gobierno chileno fue remarcar que la opción de apelar a un tribunal internacional iba en desmedro del avance del diálogo y la construcción de la confianza mutua. El giro de la política boliviana el 2012 generó un cambio en la trayectoria de la relación bilateral.

¿Cuáles fueron las motivaciones del Gobierno boliviano para romper la dinámica bilateral reflejada en la Agenda de los Trece Puntos y optar por la idea de plantear la 
intervención de los tribunales internacionales? Este es un primer aspecto que cambia el foco desde el institucionalismo que forjaron los gobiernos de la Concertación hacia una diplomacia presidencial, cuya vocería está en la palabra del canciller de Chile y por cierto, en la del canciller de Bolivia, Alfredo Moreno y David Choquehuanca, respectivamente.

Uno de los rasgos característicos de la política exterior chilena es la defensa de sus intereses territoriales y económicos, el fuerte apego a la ley y a los tratados internacionales (Valdivieso 2007). Desde una posición legalista, Chile ha manifestado su profunda vocación por el respeto a las normas internacionales y el mantenimiento de los tratados suscritos, en lo que a demandas de revisión de sus límites se refiere. Desde una perspectiva chilena, es que a partir de los tratados suscritos no existen problemas pendientes con Bolivia. Ello determina que la firma del tratado de 1904 zanja los temas territoriales y de libre tránsito, y por ello no existen conflictos entre ambos países. En el caso de Bolivia, la salida soberana al océano Pacífico es considerada como un derecho irrenunciable e imprescriptible, y en palabras del presidente Morales como "la reparación a una injusticia histórica” (La Nación 2011) y fue incluido en la Constitución del Estado Plurinacional de Bolivia (2009) y forma parte de las prioridades de su política exterior (Arts. 267-268).

Para Chile, la creciente institucionalización de su relación con Bolivia tiene como objetivo primordial la construcción de la confianza mutua, el desarrollo de iniciativas conjuntas en temas estratégicos y en términos generales, tener una relación cordial con su vecino. Para Bolivia el acercamiento y la configuración de instancias de cooperación bilateral tienen sentido en la medida en que sean un paso de avance para el logro de una respuesta concreta a su reivindicación marítima. Dado que tras cinco años del establecimiento de la Agenda de los Trece Puntos, dicha respuesta no llegó, o no por lo menos en los términos en los que el Gobierno boliviano lo ha demandado, desde la perspectiva de este país hay un agotamiento de la agenda como mecanismo y del diálogo como estrategia.

En este sentido, cabe destacar que los esfuerzos de Bolivia por lograr una salida al océano Pacífico se han estructurado desde una lógica pendular, que oscila entre las acciones encaminadas hacia el posicionamiento de la tesis del revisionismo del Tratado de 1904 en espacios multilaterales, tanto mundiales (Sociedad de las Naciones, Organización de Naciones Unidas), como interamericanos, por un lado; y por el otro, la estructuración de un despliegue diplomático para lograr un acercamiento y negociación directa con Chile. Para ello, Bolivia ha recurrido tanto a la intermediación de terceros países como a gestiones diplomáticas. 
En ese marco, la creación de la Dirección de Reivindicación Marítima (2011) apunta hacia una sistematización en dos sentidos: el primero es a buscar cualquier resquicio que permita impugnar el cumplimiento del tratado por parte de Chile y un segundo aspecto, es el que permita encontrar argumentos para enviar la situación de "enclaustramiento", a propósito del cumplimiento del mismo por parte de Chile a tribunales internacionales.

En ambos temas, la situación es compleja para Chile. Límites, libre tránsito y libertad de acceso hacia los puertos son puntos focales del reclamo del Gobierno boliviano. En relación a los límites, la discusión está cerrada en el tratado mismo; pero en la lectura de Bolivia, si los aspectos complementarios, libre tránsito y libertad de acceso a los puertos se ven entorpecidos, entonces tendría argumentos para impugnar lo suscrito. ¿Cuál es el panorama actual en estos aspectos complementarios? Cobros por almacenaje de carga boliviana en recintos concesionados, así como por la carga en tránsito desde y hacia Bolivia, requieren de consensos. Otros inconvenientes se relacionan con la carga en tránsito hacia Bolivia, que por disposiciones de la Aduana Nacional Boliviana, son impedidas de ingresar a su territorio: vehículos usados y cargas peligrosas para el medioambiente como cenizas de sodio (La Nación 2011), lo que ha redundado en una acumulación de mercaderías que dificultan su almacenaje y provocan gastos y perjuicios a las empresas portuarias que están en el puerto de Arica fundamentalmente. Es en este campo, que la demanda por la soberanía también hace eco, toda vez que Bolivia se siente que tiene que efectuar consultas permanentes en relación a su comercio exterior, asunto que, en rigor, ocurriría en cualquier puerto competitivo internacionalmente.

La infraestructura en materia de carreteras y caminos y el ferrocarril son los dos aspectos claves en el libre tránsito. Respecto a lo primero, las carreteras que unen a los dos países forman parte del acuerdo trilateral Corredor Bioceánico, que conecta a los océanos Pacífico y Atlántico a través de las carreteras de Chile, Bolivia y Brasil. Actualmente, existen pasos fronterizos integrados que facilitan el desplazamiento entre los dos países, a saber: Pasos Visviri y Chungará-Tambo Quemado en la región de Arica-Parinacota, paso Colchane-Pisiga en la región de Tarapacá y paso Salar de Ollagüe y Portazuelo de Cajón en la región de Antofagasta.

Por otra parte, en lo concerniente al Ferrocarril Arica-La Paz (FCALP), este forma parte del Tratado de Paz y Amistad de 1904 firmado por Chile y Bolivia. Desde 1928, la administración del ferrocarril quedó separada en dos sectores: de Arica a Visviri bajo control chileno, y el de Charaña a La Paz pasó a depender de Bolivia. Este funcionó hasta el 2001, cuando resultó seriamente afectado por las lluvias que azotaron la región de 
Parinacota. Entre el 2001 y 2002 se adelantaron trabajos para la reparación de las vías. Sin embargo, debido a la inactividad, la empresa que tenía a cargo la concesión de la operación del ferrocarril se declaró en quiebra en el 2005. Durante el 2009, la Empresa de Ferrocarriles del Estado (EFE) llamó a una licitación para la rehabilitación física y remediación ambiental de Ferrocarril Arica-La Paz en el tramo correspondiente al territorio chileno. Por su parte, la Empresa Portuaria de Arica EPA tenía el mandato para desarrollar el proyecto de rehabilitación física y remediación ambiental de la sección chilena durante 2010 y 2011.

En mayo de 2010, la empresa chilena Portuaria Arica y el consorcio Comsa S.A. firmaron un acuerdo para la rehabilitación de 206 kilómetros del ferrocarril (Los Tiempos 2010). Este proyecto contó con un presupuesto de 32 millones de dólares, en donde la rehabilitación física comprende 205 kilómetros de la sección chilena, el cambio de 8.000 rieles, 48.000 durmientes y sistemas de anclaje de las vías. Esto mejora ampliamente el tiempo de transferencia de carga, a una velocidad compatible con el uso comercial. Las obras concluyeron en diciembre de 2012.

En lo concerniente al acceso a los puertos chilenos, este es uno de los aspectos que mayores complicaciones ha traído a la relación vecinal. El Tratado de 1904 señala:

Artículo VI. La República de Chile reconoce en favor de la de Bolivia, y a perpetuidad, el más amplio y libre derecho de tránsito comercial por su territorio y puertos del Pacífico. Artículo VII. La República de Bolivia tendrá el derecho de constituir agencias aduaneras en los puertos que designe para hacer su comercio. Por ahora se señala por tales puertos habilitados para su comercio, los de Antofagasta y Arica (Dirección de Fronteras y Límites 2012c).

La producción boliviana se moviliza a través de cuatro puertos: Arica, Iquique, Antofagasta y Matarani (Perú). Con base en los datos entregados por la Empresa Portuaria de Arica, el puerto de Arica mantiene su liderazgo en la transferencia de carga boliviana. Entre los puertos del norte de Chile y sur de Perú fueron movilizadas 1.782.765 toneladas en el 2008, de ello 68 por ciento se hizo por el terminal ariqueño (EPA 2011). Este fue 8 puntos porcentuales superior, registrando un incremento de 21 por ciento del tonelaje transferido en comparación con el año anterior. Durante el 2010, se registró un incremento de 20 por ciento en la transferencia de carga, cerrando el año con 2.131.367 toneladas movilizadas, representando 73 por ciento de la carga que pasa por este puerto. 
En el tema portuario, las tarifas han sido la manzana de la discordia entre los dos países. En el 2004, el puerto de Arica fue dado en concesión a la Empresa Portuaria de Arica. Ello despertó la preocupación del Gobierno boliviano respecto a un eventual aumento tarifario y a la intermediación de privados en un asunto que forma parte de las condiciones del Tratado de 1904. Así, en octubre de 2004, una misión del Gobierno boliviano viajó a Chile para discutir la legalidad de la concesión a privados del puerto, a la que el Gobierno boliviano se mostró contraria (Radio Cooperativa 2004). En este mismo mes, el entonces presidente de Bolivia, Carlos Mesa, anunció en una entrevista televisada que recurriría a los tribunales superiores de justicia si Chile no garantizaba el comercio boliviano a través del puerto (América Economía 2004), y señaló la intención de buscar nuevas opciones portuarias para Bolivia, como el puerto de Matarani en Perú. La principal preocupación de las autoridades bolivianas tras la privatización del puerto era el aumento de las tarifas, estimado en 250 por ciento. A ello, el presidente chileno, Ricardo Lagos, respondió que el mayor costo se vería compensado con la mayor eficiencia en la operación (Infobae 2004). Cabe aclarar que virtud del Tratado de 1904, la mercancía boliviana tiene tratamiento especial en los puertos de Arica, Iquique y Antofagasta. Por ejemplo, para el almacenamiento de la carga, Bolivia cuenta con 365 días liberados del pago del Servicio de Almacenamiento para las cargas de importación y de 60 días para las cargas de exportación. Según estimaciones del 2004, el costo para el Fisco de Chile que implica la gratuidad en el almacenamiento de la carga boliviana es de aproximadamente \$2.500 millones al año, más de US\$ 450 millones (Contexto.org 2004).

En el 2007, nuevamente las autoridades bolivianas protestaron frente al aumento de las tarifas portuarias. El entonces canciller boliviano, Mauricio Dolfler, fue enfático al señalar que Bolivia no reconocía a la empresa Terminal Puerto Arica como intermediaria entre el Estado y los empresarios bolivianos (La Prensa 2007). La más reciente de las polémicas en torno a las tarifas se dio en septiembre de 2011 y surge cuando la entonces directora de la Administración de Servicios Portuarios de Bolivia (ASPB), Marianela Prada, afirmó que el Gobierno boliviano rechazaba cualquier tipo de incremento unilateral en las tarifas portuarias en tanto no haya una respuesta y solución definitiva a la demanda marítima. En sus palabras: "Hasta que este problema estructural y de fondo que hace que nuestro libre tránsito no sea resuelto" (América Economía 2011). Días después, en el marco del Grupo de Libre Tránsito, el Gobierno de Bolivia solicitó a Chile administrar de forma directa el puerto de Arica. El tema tratado en la reunión del mecanismo de consultas políticas programada para finales de 2011, ha dado como resultado que el vicecanciller de Bolivia, Juan Carlos Alurralde, señale: "Tenemos el temor de que EPA transfiera el ferrocarril a la TPA y eso sería consolidar un monopolio que nos está asfixiando", 
puntualizó, y aseguró que 90 por ciento de las exportaciones bolivianas a ultramar salen precisamente por el puerto de Arica (MDZ 2011).

No obstante, las opiniones hay que colocarlas en su contexto económico. De acuerdo a los datos proporcionados por el Instituto Boliviano de Comercio Exterior, la carga boliviana no tradicional casi se ha duplicado en los últimos seis años. Esto nos lleva a preguntarnos seriamente si Chile cumple o no con las condiciones portuarias para la exportación que demanda Bolivia. Resulta de utilidad saber que los mecanismos de exportación tramitados por Bolivia deben cumplir con aspectos convencionales de los despachos aduaneros y de carga. Estos son comunes a todos los puertos del país, con el propósito de cumplir con los resguardos comerciales para el control del narcotráfico.

Con todo, Bolivia tiene un desafío adicional, cual es su propio manejo sobre la carga que sale y entra por Chile hacia y desde Bolivia. La Administradora de Servicios Portuarios de Bolivia (ASPB) intermedia en el transporte de la carga que sale por el Puerto de Arica; no ha sido nunca el Terminal Puerto Arica (TPA). Es aquella la que cobra un monto por salida y entrada de mercancías, en una "caja negra", sin estándares de los cobros, o una página web transparente con las tarifas o funciones. En efecto, la Administradora de Servicios Portuarios de Bolivia es el único cliente de Bolivia en el puerto de Arica, por lo que el Terminal Puerto Arica tarifa de manera directa con la Administradora y no con los clientes. Esto implica que Chile no pone trabas a los exportadores, sino que se entiende - por mandato boliviano- con una sola institución para toda la carga en tránsito. Con esto, los bolivianos, antes de reclamar a la Empresa Portuaria de Arica, que fiscaliza la carga o con el Terminal Puerto Arica, que es la empresa que tiene desde el 2004 la concesión del Puerto, debería revisar por qué la Administradora de Servicios Portuarios de Bolivia cobra adicionalmente por el valor de salida y entrada de la carga cuando esta entra o sale de la propia Bolivia. 


\section{Grafico $\mathbf{N}^{\circ} 1$}

Bolivia: exportaciones según valor

Gestiones 2005-2010 y avance a noviembre de 2011 (p)

(En millones de dólares americanos)

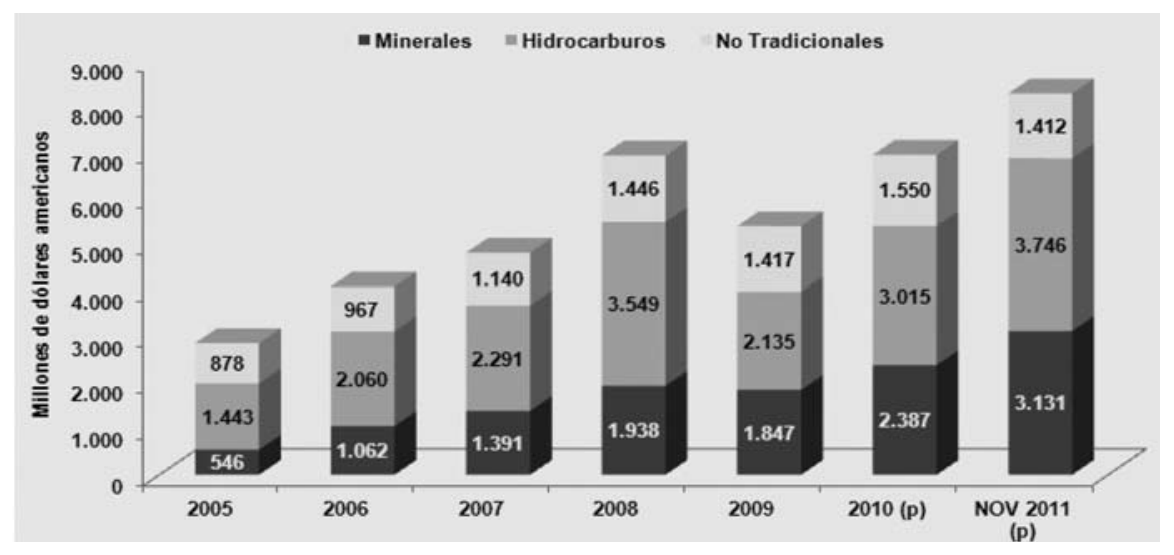

Fuente: Instituto Boliviano de Comercio Exterior. Boletín No 25 de mayo de 2012.

$\mathrm{Al}$ respecto, resulta ilustrador saber que la Empresa Portuaria Arica y la Administración de Servicios Portuarios de Bolivia (ASPB) firmaron la tercera acta de entendimiento tarifario, período 2012-2013, por los servicios prestados en el puerto de Arica para las cargas en tránsito desde y hacia Bolivia.

La crítica boliviana se desmorona al entender que el Terminal Puerto Arica se ha constituido en el puerto con las tarifas más competitivas del norte chileno, y consolidado como salida natural para las cargas de Bolivia. Por ello:

Este acuerdo establece las tarifas especiales a los servicios que TPA le presta a los clientes bolivianos que operan a través de ASPB y estamos plenamente satisfechos porque nos permite seguir trabajando en el desarrollo de la cadena logística. El libre tránsito es una materia inherente a los servicios de nuestro puerto y reafirmamos con este convenio nuestra vocación por atender con eficiencia y competitividad la carga boliviana..., [puntualiza el presidente de Puerto Arica]. (Mundo Marítimo 2012).

¿Qué explica la contradicción entre lo dicho por el Estado boliviano y la praxis portuaria? El hecho de que Bolivia no acepta la privatización de los puertos, factor sobre el cual Chile no ofrece alternativa. 
En relación al reclamo por el tren, Chile licitó el tren de Arica a La Paz en 1997. El 2004, el Ferrocarril de Arica a La Paz (FCALP), cuyo tramo chileno fue entregado al empresario boliviano José Saavedra Bánzer, intentó pedir ayuda al grupo Luksic. Sin embargo, el estado de la empresa hizo inviable el negocio (Silva 2005).

Es cierto que el tren no opera, y no lo hace porque la empresa que se adjudicó la concesión del tren quebró. Lo que no maneja la opinión pública generalizada en Bolivia es que a diferencia del Terminal Puerto Arica, consorcio de cinco empresas -donde una de ellas es peruana-, en el caso del tren, la empresa era boliviana. Por ello, la imputación de la falta de atención por parte de Chile es incorrecta. Es cierto, el tiempo ha transcurrido, pero ha llevado a que hoy se hayan invertido no 26 millones de dólares como apuntaba el proyecto, sino 32 millones de dólares en la recuperación de la vía que movilizará cerca de 100.000 toneladas más de carga boliviana al año. Las operaciones repondrán una línea férrea a la no poca cantidad de 4.000 metros de altura en algunos tramos.

\section{Tabla $\mathbf{N}^{\circ} 2$}

Importaciones bolivianas de acuerdo al puerto de ingreso en Chile en millones de toneladas

\begin{tabular}{|c|c|c|c|}
\hline Años & $\begin{array}{c}\text { Arica/ Charaña-Tambo } \\
\text { Quemado }\end{array}$ & $\begin{array}{c}\text { Iquique /Pisiga-Bella } \\
\text { Vista }\end{array}$ & Antofagasta/Ollagüe-Uyuni \\
\hline 2008 & 909.984 & 146.436 & 86.326 \\
\hline 2009 & 852.610 & 117.908 & 61.429 \\
\hline 2010 & 1.143 .705 & 117.922 & 82.488 \\
\hline 2011 & 1.602 .519 & 157.753 & 86.813 \\
\hline 2012 & 1.530 .076 & 202.521 & 79.779 \\
\hline Total & 6.038 .894 & 742.540 & 396.835 \\
\hline
\end{tabular}

Fuente: Elaboración propia en base a cifras del IBCE 2008-2012.

Chile reconoce el beneficio que el comercio y el libre tránsito representa para Arica. En atención a la envergadura de los beneficios económicos que el libre tránsito representa para la carga de Bolivia, entre 60 y 70 por ciento, entre el 2008 y 2010, el tema es relevante para Bolivia, pero también para Arica y Parinacota.

Sin embargo, desde la perspectiva boliviana, el tema marítimo supera las consideraciones geoestratégicas o económicas. La visión de su mediterraneidad como producto de una injusticia histórica y la legitimidad de su reclamo de una salida soberana al Pací- 
fico forman parte del imaginario de Bolivia como nación, es decir, excede el ámbito de la interacción estratégica.

\section{CONCLUSIONES}

Durante el siglo XX, la relación de Chile con Bolivia se caracterizó por la conflictividad y desconfianza. Ante la ausencia de un cierre definitivo para el tema de la mediterraneidad boliviana y su demanda por una salida soberana al océano Pacífico, los dos países mantienen rotas sus relaciones diplomáticas. No obstante, desde la década de 1990, temáticas como el comercio, seguridad y migración, llevaron a un acercamiento entre los dos países y la creciente institucionalización de la relación bilateral.

La mayor interdependencia, la transición democrática en Chile, el desarrollo de un modelo económico basado en la apertura comercial por parte del Gobierno boliviano y el contexto regional, son algunos de los factores que han servido de incentivo para el cambio en la trayectoria de la relación. Sin embargo, la relación fue de menos a más y de más a cero. Su pick se desarrolló con la administración Bachelet y Morales.

La Agenda de los Trece Puntos fue el resultado de la creciente interacción estratégica, constituyendo la hoja de ruta en la relación bilateral y el mecanismo para la resolución de los asuntos bilaterales mediante el diálogo. La cuestión marítima y de recursos hídricos subsisten como el principal desacuerdo entre los dos países, con posiciones contrarias e irreductibles. La petición del Gobierno boliviano de administrar de forma directa el puerto de Arica -septiembre de 2011- abrió una nueva fisura, y evidencia una absoluta voluntad boliviana por mantener el reclamo del territorio soberano al norte de Arica, como estrategia de fondo. La inclusión del tema marítimo en la Constitución del Estado Plurinacional de Bolivia (2009) demuestra la reinstalación del reclamo por acceso soberano al mar de manera permanente en la agenda exterior de Bolivia, lo cual, sumado a la creación de la Dirección de Reivindicación Marítima en 2011, evidencia que hay una nueva oscilación en el péndulo de los esfuerzos de Bolivia por lograr una salida al océano Pacífico, pasando desde un despliegue diplomático para lograr un acercamiento y negociación directa con Chile, hacia el posicionamiento de la tesis del revisionismo del Tratado de 1904 en un escenario internacional. Hoy, en su momento más bajo de la relación vecinal, la política exterior realista ha confirmado ser nacionalista, presidencialista e ineficaz en ambos países. 


\section{REFERENCIAS}

1. América Economía. 2004. "Bolivia amenaza a Chile con recurrir a los Tribunales superiores." Madrid: Asesores de Publicaciones Economía. Consultado 10 de agosto de 2012. (http:// www.americaeconomica.com/numeros4/288/noticias/vboliviachilelu.htm)

2_ 2011. "Bolivia: acusan que Chile impone restricciones al Comercio Exterior." Argentina: América Economía, 07 de septiembre. (http://www.americaeconomia.com/economiamercados/comercio/acusan-que-chile-impone-restricciones-al-comercio-exterior-de-bolivia)

3. Comisión Nacional de Desminado. 2010. "Ministros de Defensa de Chile y Bolivia declaran libres de minas terrenos en Tambo Quemado." Santiago: Estado Mayor Conjunto. Consultado 31 de julio 2012 (http://www.cnad.cl/sitio/noticias/2010/07/310710.html)

4. Correa, Loreto. 2007. "El proceso de integración cultural entre Chile y Bolivia: desde su historia, sus perspectivas y desafíos." Pp. 257-82 en Nuestros vecinos, editado por M. Artaza y P. Milet. Santiago: RIL Editores.

5. Contexto.org. 2004. "Las relaciones chileno-bolivianas en el centenario del Tratado de Paz y Amistad de 1904." Contexto.org, Bolivia. Consultado 06 de julio 2012 (http://www.contexto. org/pdfs/chile_bolivia.pdf)

6. Del Arenal, Celestino. 1994. Introducción a las relaciones internacionales. Madrid: Tecnos.

7. Deustua, Alejandro. 2004. "Perú, Bolivia y Chile: por una nueva relación trilateral." Revista de Ciencia Politica XXIV (2):212-27.

8. Dirección de Fronteras y Límites (DIFROL). 2012a. Paso Visviri. Santiago: MINREL. Consultado 12 de julio 2012 (http://www.difrol.cl/index.php?option=com_content\&task=view\& $\mathrm{id}=176 \&$ Itemid $=10)$.

9._. 2012b. Paso Colchane-Pisiga. Santiago: MINREL. Consultado 12 de julio 2012 (http:// www.difrol.cl/index.php?option=com_content\&task=view\&id=54\&Itemid=10).

10. - 2012c. Tratado de Paz, Amistad y Comercio entre Chile y Bolivia, 1904. Santiago: DIFROL. Consultado 10 de septiembre 2012 (http://www.difrol.cl/index.php?option=com_con tent\&task=view\&id=26\&Itemid $=12$ )

11. El Deber. 2006. "Encuentro entre presidentes de Bolivia y Chile." El Deber, Bolivia 28 de enero. 
12. Empresa Portuaria de Arica (EPA). 2011. Memorias puerto Arica 2011. Arica: EPA. Consultado 05 de agosto de 2012 (http://www.puertoarica.cl/Web/memorias.html).

13. Fazio, Hugo. 1997. “Chile: Modelo de desarrollo e inserción internacional." Historia crítica 13: 68-89.

14. Infobae. 2004. “Mesa y Lagos ratifican diferencias por conflicto limítrofe.” Argentina: Infobae. Consultado 12 de agosto 2012 (http://www.infobae.com/notas/nota.php?Idx=146981\&I$\mathrm{dxSeccion}=0)$.

15. Keohane, Robert. 1993. Instituciones internacionales y poder estatal: ensayos sobre teoría de las relaciones internacionales. Buenos Aires: Grupo Editor Latinoamericano.

16. La Nación. 2011. "Evo Morales reivindica demanda marítima ante la ONU.” La Nación, Santiago 21 de septiembre. Consultado 10 de agosto 2012 (http://www.lanacion.cl/evo-morales-reivindica-demanda-maritima-ante-la-onu/noticias/2011-09-21/214656.html).

17. La Razón. 2006a. “Un encuentro histórico entre Lagos y Morales.” La Razón, Bolivia 20 de enero.

18._ 2006b. "Bolivia y Chile cuidan no enturbiar la nueva relación.” La Razón, Bolivia, 22 de febrero.

19. La Prensa. 2007. “Chile asegura que Cancillería avaló la privatización en Arica." La Prensa, Bolivia, 15 de febrero.

20. La Voz. 2006a. "Evo y Michelle Bachelet buscarán entendimiento." La Voz, Bolivia 7 de marzo.

21._. 2006b. "Evo prioriza lazos con Chile." La Voz, Bolivia, 23 de febrero.

22. Los Tiempos. 2006. “La visita de Lagos a Bolivia será histórica.” Los Tiempos, Bolivia, 18 de enero. Consultado 10 de agosto 2012. (http://www.lostiempos.com/diario/actualidad/nacional/20060118/la-visita-de-lagos-a-bolivia-sera-historica_2233_2233.html)

23._ 2010. "Bolivia y Chile retoman ferrocarril Arica-La Paz." Los Tiempos, Bolivia 02 de mayo.

24. Ministerio de Defensa. 2010a. "Chile y Bolivia firmaron Acuerdo de Cooperación en Defensa." Santiago: noticias Ministerio de Defensa. Consultado 22 de junio 2012. (http://www. defensa.cl/noticias/chile-y-bolivia-firmaron-acuerdo-de-cooperacion-en-defensa) 
25. Ministerio de Relaciones Exteriores, Sala de Prensa. 2010a. "Comisión Mixta sobre Drogas Chile-Bolivia termina con importante acuerdo.” Santiago: MINREL. Consultado 25 de agosto 2012. (http://www.minrel.gob.cl/prontus_minrel/site/artic/20100825/pags/20100825081548. php).

26. 2010b. Acta de la XXII reunión del mecanismo de consultas políticas Bolivia-Chile. Santiago: MINREL. Consultado julio 2012. (http://www.minrel.gob.cl/prontus_minrel/site/ artic/20100715/asocfile/20100715165143/acta_final.pdf).

27. MDZ. 2011. "Bolivia le promete a Chile una 'agresiva política' de salida al mar.” Mendoza: MDZ, 20 de noviembre. Consultado 11 de julio 2012. (http://www.mdzol.com/mdz/mobile/342875/)

28. Mundo Marítimo. 2012. "Empresa Portuaria Arica y ASPB alcanzan acuerdo tarifario 2012-2013." Chile: Mundo Marítimo, 09 de abril. Consultado 11 de julio 2012. (http:// www.mundomaritimo.cl/noticias/empresa-portuaria-arica-y-aspb-alcanzan-acuerdo-tarifario-2012-2013)

29. Muriel, Beatriz y Gover Barja. 2006. Inserción internacional en Bolivia: Estrategias, resultados y perspectivas. Documento de Trabajo. La Paz: Universidad Católica Boliviana.

30. Orias, Ramiro. 2002. "La diplomacia del gas boliviano: integración energética y geopolítica en la región." Estudios Internacionales 35 (138):151-71.

31. ProChile, 2009. “Informe Comercio Exterior Chile-Bolivia 2009." Santiago: Direcon. Consultado 18 de mayo 2010 (http://rc.direcon.cl/sites/rc.direcon.cl/files/bibliotecas/COMERCIO_EXTERIOR_BOLIVIA_2009.pdf).

32. Quezada, Abraham. 2010. “Inserción internacional de Chile en la post guerra fría.” Enfoques VIII (13):119-34.

33. Radio Cooperativa. 2004. "Misión boliviana viajará el jueves a Arica para discutir sobre concesión portuaria." Santiago: Radio Cooperativa. Consultado 11 de julio 2012. (http:// www.cooperativa.cl/noticias/pais/relaciones-exteriores/bolivia/mision-boliviana-viajara-eljueves-a-arica-para-discutir-sobre-concesion-portuaria/2004-10-04/212841.html)

34. Seoane, Alfredo. 2008. "La inserción de Bolivia en la economía mundial. Una mirada crítica al post-neoliberalismo." Umbrales 17:57-92.

35. Silva, Mauricio. 2005. "En quiebra se declara el Ferrocarril de Arica a La Paz." El Mercurio, 14 de diciembre. 
36. Vaca, Mery. 2008. "Bolivia/Chile: Acuerdo de defensa." BBC Mundo, 17 de junio. Bolivia. Consultado 11 de agosto 2012. (http://news.bbc.co.uk/hi/spanish/latin_america/ newsid_7458000/7458214.stm)

37. Valdivieso, Patricio. 2007. "A propósito de las relaciones Chile-Bolivia-Perú: Percepciones, experiencias y propuestas.” Bicentenario, Revista de Historia de Chile y América 6 (2):99-123.

38. Wilhelmy, Manfred y Roberto Durán. 2003. "Los principales rasgos de la política exterior chilena entre 1973 y el 2000." Revista de Ciencia Política XXIII (2):273-86. 